\title{
Asertividad: definiciones y dimensiones
}

\section{Vicente E. Caballo}

\author{
Universidad Autónoma de Madrid
}

El término "asertividad» empleado de manera tan frecuente en la década pasa$\mathrm{da}$, es todavía un constructo que no tiene una definición universalmente aceptada. Se puede decir que en general hay un acuerdo tácito sobre lo que representa aproximadamente el vocabo "asertividad». Pero algunos autores, y sobre todo a nivel popular, le dan un sentido limitado o le confunden con otro constructo como es la «agresividad». En los Estados Unidos, por ejemplo, el constructo de la «asertividad» es confundido popularmente, según Rathus (1979), con la agresividad y está asociado para muchos con el atrevimiento social (confundido con beligerancia, obscenidad y mala fe).

El término mismo de "asertividad» ha sufrido numerosos ataques. Muchas veces se ha intentado su sustitución por otros vocablos. Ya desde el comienzo de su expansión, a principios de los años setenta, uno de sus creadores y difusores propone el cambio del término "asertividad" por el de «libertad emocional" (Lazarus, 1971), considerado más conveniente. La misma fuente de donde Wolpe extrae el concepto de la "asertividad", vocablo usado por primera vez en su libro «Psicoterapia por inhibición recípro- ca» en 1958, emplea el término "personalidad excitatoria" (Salter, 1949) y no el de "conducta asertiva" utilizado posteriormente por Wolpe y sus colaboradores. Ya a mediados de los años setenta se barajan otros términos sustitutorios como "competencia conductual", "efectividad personal", etc. Heimberg y cols. (1977) sugieren de igual manera el empleo de la expresión "competencia social». Pero el término que realmente está desplazando hoy en dia al de la (asertividad" y que probablemente acabará sustituyéndolo es el de "habilidades sociales». Este último término se puede ver actualmente en numerosos estudios clíni$\cos$ y experimentales. Hay autores, sin embargo, que reducen el significado de la "asertividad» y la consideran tan sólo como un tipo de «habilidad social» (Matson y cols., 1980; Bornstein y cols., 1977; Romano y Bellack, 1980). Trower, Bryant y Argyle (1978) señalan, no obstante, que la diferencia que existe entre el término "asertividad» y el de «habilidades sociales", tal y como ambos se consideran hoy día, es más bien resultado de un accidente histórico. Tengo que señalar además que con los términos «entrenamiento asertivo» (Alberti, 1977a, 
1977b; Alberti y Emmons, 1978; Fensterheim y Baer, 1976; Kelley, 1979; Smith, 1977) o uentrenamiento en habilidades sociales" (Bellack, 1979; Curran, 1977 [nota 1], 1979; Trower, 1979; Trower y cols., 1978) se designan prácticamente el mismo conjunto de procedimientos de tratamiento y el mismo grupo de dimensiones conductuales a entrenar.

\section{LAS RAICES}

El concepto de la asertivididad tiene sus raices en la terapia de conducta, sobre todo en el trabajo de Andrew Salter, Joseph Wolpe y Arnold Lazarus. El primero en usar la palabra «aserción» es Wolpe en su libro «Psicoterapia por inhibición reciproca» en el año 1958, como se dijo anteriormente. Aunque años antes A. Salter (1949) habia ya establecido las bases para una posterior teoría y práctica de la asertividad al describir su modelo «excitatorio». Posteriormente al libro de Wolpe citado anteriormente, no es sino hasta mediados de los años sesenta que aparece el «entrenamiento asertivo" como una técnica más de la terapia de conducta, en el libro de J. Wolpe y A. Lazarus, "Técnicas de terapia de conducta» en el año 1966 o comparando el ensayo de conducta (parte básica y esencial del entrenamiento asertivo) con otras técnicas de tratamiento (Lazarus, 1966). En el año 1970 aparece el primer libro dedicado exclusivamente al tema de la asertividad, el libro de Alberti y Emmons (1978) en su primera edición de 1970 . Y es a partir de los primeros años setenta que el constructo de la asertividad adquiere cada vez mayor expansión $y$ popularidad $y$, a mediados de la década de los setenta, alcanza tal repercusión que se forman asociaciones dedicadas exclusivamente al progreso del entrenamiento asertivo, se le llega a considerar un tipo de tratamiento ideal para prácticamente todo tipo de problema de relación social, se intenta desvincularlo de la terapia de conducta, y se crea una revista dedicada exclusivamente al tema de la asertividad ( $A$ ASSERT: The Newsletter of Assertive Behaviors), aparte de los innumerables libros y artículos que se publican en esa época, todo ello en los Estados Unidos.

Hoy en día el término de la wasertividad» va perdiendo fuerza entre los profesionales a pasos agigantados, como he señalado anteriormente, aunque siga conservando (junto con muchas confusiones y errores sobre el concepto) gran popularidad en los Estados Unidos. La expresión «habilidades sociales» se va imponiendo en su lugar como un vocablo más idóneo y más cercano a la realidad que abarca. En España es posible que el término «asertividad» sea algo más conocido que el de «habilidades sociales» debido fundamentalmente a dos libros de corte popular editados en español (Fensterheim y Baer, 1976; Smith, 1977) y a algunos anuncios sobre grupos de entrenamiento asertivo dirigidos a personas con problemas de relaciones interpersonales. No obstante no se puede decir que el término "asertividad» sea conocido popularmente en España, por lo que un cambio de ese término por el de «habilidades sociales» no supondría ningún objetivo prioritario. Serían los profesionales los que tendrian que inclinarse por uno u otro término, aunque en este sentido una tercera expresión como la de «competencia social» podría también entrar en danza.

\section{DEFINICIONES}

Pero, ¿qué es la conducta asertiva? Si miramos en el diccionario la palabra «aserción» es sinónimo de "afirmación». $Y$ aunque la afirmación de los derechos de uno mismo entraría dentro del concepto de la "asertividad», no se puede considerar el significado que se encuentra en el diccionario de la palabra uaserción» como equivalente a ella. Este vocablo tiene una significación muy amplia y abarca una gran diversidad de conductas. 
Por otra parte no hay una definición universalmente aceptada de la conducta asertiva. No obstante se ha tratado de definirla en numerosas ocasiones. Algunas más acertadas que otras, se han escogido una serie de definiciones que pueden ser útiles a la hora de dar con una definitiva.

Para Alberti y Emmons (1978) la conducta asertiva es:

"La conducta que permite a una persona actuar en base a sus intereses más importantes, defenderse sin ansiedad inapropiada, expresar cómodamente sentimientos honestos o ejercer los derechos personales sin negar los derechos de los otros" (p. 2).

Para Brown (1980) es:

"La conducta que ocurre en un contexto interpersonal que se interesa principalmente por la adquisición de refuerzo personal y que minimiza la pérdida de refuerzo por parte del (los) receptor(es) de $\mathrm{la}(\mathrm{s})$ conducta(s)» (p. 265).

Libet y Lewinshon (1973), recogido por Galassi y Galassi (1979) señalan que es:

"La capacidad compleja de emitir conductas que son reforzadas positivamente o negativamente, y de no emitir conductas que son castigadas o extinguidas por los demás."

McDonald (1978) define la aserción como:

«La expresión abierta de las preferencias (por medio de palabras o acciones) de una manera tal que haga que los otros las tomen en cuenta» (p. 889).

Rich y Schroeder (1976) definen la conducta asertiva como:

«La habilidad de buscar, mantener o mejorar el reforzamiento en una situación interpersonal a través de la expresión de sentimientos o deseos cuando esa expresión se arriesga a la pérdida de reforzamiento $o$ incluso al castigon ( $p$. 1082).

Para Rimm y Master (1974) es:

"La conducta interpersonal que implica la honesta y relativamente directa expresión de sentimientos» (p. 81).

Para Wolpe (1977) es:
«La expresión adecuada dirigida hacia otra persona de cualquier emoción que no sea la respuesta de ansiedad" (p. 96).

Un grupo de psicólogos y educadores (Alberti y otros, 1977) se reunieron en 1976 y establecieron unos «Principios para la práctica ética del entrenamiento asertivon. Dentro de estos principios proponen la siguiente definición de la conducta asertiva:

"Se define la conducta asertiva como ese conjunto de conductas, emitidas por una persona en un contexto interpersonal, que expresa los sentimientos, actitudes, deseos, opiniones o derechos de esa persona de un modo directo, firme y honesto, respetando al mismo tiempo los sentimientos, actitudes, deseos, opiniones y derechos de la(s) otra(s) persona(s). Esta conducta puede incluir la expresión de emociones tales como ira, miedo, aprecio, esperanza, alegría, desesperación, indignación, perturbación, pero en cualquier caso se expresa de una manera que no viola los derechos de los otros. La conducta asertiva se diferencia de la conducta agresiva en que ésta, mientras expresa los sentimientos, actitudes, deseos, opiniones o derechos de una persona, no respeta estas características en los otros») (p. 367-368).

De todas estas definiciones hay algunas que enfatizan la autoexpresión de uno mismo sin herir a los demás, y puede que ésta sea la característica común que deben llevar todas las definiciones de la conducta asertiva. Sin embargo otras definiciones insisten principalmente en la efectividad de esa conducta, en la consecución de los objetivos propuestos. Parece ser que está fuera de duda, entre los estudiosos de la asertividad, que la característica común que he señalado antes está implícita en toda conducta asertiva. Pero ¿puede considerarse conducta asertiva un comportamiento que no es reforzado positivamente o que no alcanza su objetivo? Hay autores que opinan que sí, que el alcanzar al objetivo externo no es fundamental (Alberti y Emmons, 1978), mientras se autoexpresa el individuo responsablemente, mientras 
que otros autores (Eisler y Frederiksen, 1980) consideran esencial la consecución del objetivo. De igual manera Warren y Gilner (1978) señalan que las conductas asertivas buscan optimizar el reforzamiento en el contexto social. También Rich y Schroeder (1976) piensan que el grado de asertividad puede medirse por la efectividad de las respuestas de un individuo en producir, mantener o mejorar el reforzamiento. Para estos últimos autores una respuesta puede considerarse más efectiva cuando produce reforzamiento en una situación improbable de producir reforzamiento, o en una que implica un alto riesgo de perder el reforzamiento. Esta concepción de la asertividad de una respuesta puede ser confundida a veces con una respuesta agresiva ya que la diferencia entre ambas parece ser que, aunque las dos consiguen sus objetivos a corto plazo, la respuesta agresiva no respeta a los demás. De todas formas, la respuesta agresiva es menos reforzante a largo plazo y suele provocar en los individuos que se ven sometidos a ella, sentimientos de venganza, animadversión o ira. Creo, por otra parte, que la autoexpresión responsable es reforzante en sí misma, aunque no se alcance la meta a la que se dirige la conducta. No es intrínsecamente necesaria la consecución del objetivo para que una conducta sea considerada asertiva. Sin embargo es posible que un fracaso continuado en conseguir el objetivo haga sospechar de la efectividad de esa conducta y de si realmente ese comportamiento es asertivo. Por lo que una prudente definición del comportamiento asertivo seria: la conducta asertiva es aquella conducta que expresa los sentimientos y pensamientos de un individuo de una manera honesta sin herir los de los demás, y que normalmente alcanza su objetivo.

\section{DIMENSIONES DE LA CONDUCTA ASERTIVA}

La asertividad no es un "rasgo" de las personas. El individuo no es «asertivo» o "no asertivo", sino que un individuo se comporta asertivamente o no-asertivamente en una variedad de situaciones. Un sujeto puede desarrollar una conducta asertiva en una serie de situaciones mientras que en otras su conducta puede calificarse como no-asertiva o agresiva. No obstante hay individuos cuya conducta en la gran mayoria de las situaciones suele ser no-asertiva y entonces se habla, no sin cierta generalización, de un individuo no-asertivo. Con Alberti (1977b) tengo que decir que la asertividad:

1. Es una característica de la conducta, no de las personas.

2. Es una característica especifica a la persona y a la situación, no universal.

3. Debe contemplarse en el contexto cultural del individuo, así como en términos de otras variables situacionales.

4. Está basada en la capacidad de un individuo de escoger libremente su acción.

5. Es una característica de la conducta socialmente efectiva, no dañina.

Aunque la gran mayoria de los cuestionarios diseñados para medir la asertividad tratan de hacerlo como si se tratase de una característica unitaria de las personas (salvo excepciones, como el "Cuadro de autoevaluación de la aserción» de Galassi y Galassi [1977]), lo cierto es que la asertividad se compone de varias dimensiones. Las dimensiones exactas que abarca la asertividad y que la agotan están todavia por establecer. Sin embargo a nivel práctico el entrenamiento asertivo ha tenido que considerar diferentes dimensiones a la hora de entrenar a los sujetos con problemas interpersonales. La clasificación realizada por Lazarus (1973) en base a su experiencia clínica ha sido ampliamente utilizada llegado el momento del entrenamiento con pacientes. Aunque su origen no es experimental ha habido últimamente algunos estudios que han confirmado una parte de esta multidimensionalidad de la conducta asertiva (Lorr y cols., 1979; 1980; 


\section{Estudios}

1981). Las cuatro dimensiones propuestas por Lazarus (1973) son:

a) La capacidad de decir NO

b) La capacidad de pedir favores o hacer peticiones

c) La capacidad de expresar sentimientos positivos y negativos

d) La capacidad de iniciar, continuar y terminar conversaciones generales.

Estas cuatro dimensiones conductuales parecen ser independientes unas de otras, de modo que el entrenamiento en una de ellas no influye en las otras; además la conducta de un individuo puede poseer una o varias de estas dimensiones de forma correcta, siendo deficiente en las otras dimensiones restantes. Considero esta clasificación de la conducta asertiva como la más práctica a la hora del entrenamiento asertivo y aunque su origen no es experimental parece que se acerca mucho a lo que el constructo de la asertividad comprende.

Otros autores han propuesto otras clasificaciones, que pueden o no estar incluidas en las cuatro dimensiones anteriores, pero que desde un punto de vista conceptual las sitúan a niveles diferentes. Así, por ejemplo, Galassi, Delo, Galassi y Bastien (1974) abogan por las siguientes tres dimensiones de la asertividad:

I. Asertividad positiva, que consiste en la expresión de sentimientos de amor, afecto, admiración, aprobación y estar de acuerdo.

II. Asertividad negativa, que incluye las expresiones de sentimientos justificados de ira, desacuerdo, insatisfacción y aburrimiento.

III. Autonegación, que incluye un excesivo disculparse, excesiva ansiedad interpersonal y exagerado interés por los sentimientos de los otros.

Gambrill y Riche (1975) dividieron la asertividad en las siguientes categorias:
1. Rechazo de peticiones.

2. Expresión de limitaciones personales.

3. Iniciación de contactos sociales.

4. Expresión de sentimientos positivos.

5. Manejo de las críticas.

6. Diferir con los demás.

7. Aserción en situaciones de servicio.

8. Dar retroalimentación negativa.

Liberman y cols. (1977b), citados por Curran (1979) registran las siguientes dimensiones de la conducta asertiva:

a) Conductas no verbales discretas, como el contacto ocular, la expresión facial, la rapidez del habla, la latencia de la respuesta, volumen y tono de voz, la duración de la respuesta, la fluidez de la conversación, los gestos y la postura.

b) El contenido de la conversación, como pedir algo a otra persona, alabar, agradecer o hacer cumplidos a otras personas, decir no a una petición irrazonable, sufrir una entrevista de trabajo, reaccionar apropiadamente a la crítica, y manejar otros encuentros afectivos e instrumentales diarios.

c) Reciprocidad en la comunicación, como el dar reforzamiento al otro al mantener una conversación, iniciar conversaciones, terminar conversaciones, y regular la entrada o salida de grupos sociales.

Turner y Adams (1977) consideran tres dimensiones de la asertividad:

1) Conducta de rechazo.

2) Expresión de sentimientos positivos (asertividad "comendatoria»).

3) Capacidad de adquirir la información solicitada.

Rinn y Markle (citados por Van Hassels y cols. [1979]) dividen la conducta asertiva en cuatro repertorios conductuales: 
a) Habilidades autoexpresivas.

b) Habilidades que mejoran a los otros.

c) Habilidades asertivas en un sentido restrictivo usado por algunos autores, como el hacer peticiones simples, estar en desacuerdo con la opinión del otro, negarse a peticiones irrazonables.

d) Habilidades comunicativas.

Wolpe (1977) y sus colegas dividen la conducta asertiva en dos dimensiones:

A) Conducta asertiva positiva ("comendatorias).

B) Conducta asertiva negativa («hostil»).

Lorr y cols. (1979; 1980; 1981) han hallado cuatro dimensiones que componen la estructura de la asertividad. Aunque señalan que puede haber más, parece que estos cuatro son básicos. Las dimensiones halladas son:

1. Asertividad social. Es una disposición y capacidad para iniciar, mantener $o$ terminar las interacciones sociales fácil y cómodamente, en situaciones que implican amigos o conocidos, extraños o figuras de autoridad.

2. Defensa de los derechos. Refleja la capacidad de defender los derechos propios o rechazar peticiones irrazonables.

3. Independencia. Es la disposición de resistir activamente la presión individual o de grupo para conformarse y obedecer así como para expresar las opiniones y creencias propias.

4. Liderazgo. Es la disposición para conducir, dirigir o influenciar a los otros en relaciones interpersonales problemáticas que exigen acción, iniciativa o asunción de responsabilidades.

Lorr, More y Mansueto (1981) señalan que cada dimensión de respuesta puede conceptualizarse como una disposición relativamente duradera para comportarse de maneras específicas en ciertas situaciones de estimulo comunes. Además, al parecer, estas cuatro dimensiones forman una jerarquía, siendo el orden de adquisición: asertividad social, defensa de los derechos, independencia y liderazgo.

Como se ve no hay unas dimensiones de la asertividad universalmente aceptadas, aunque la mayoría giran alrededor de las propuestas por Lazarus (1973) o las de Wolpe (1977). Las clasificaciones de ambos autores tienen un origen clinico, y aunque la clasificación de Lazarus ha sido parcialmente confirmada por algunos estudios experimentales, se necesita una mayor investigación sobre el tema.

Finalmente, y enlazando con el comienzo de este apartado, tengo que señalar la especificidad situacional de la conducta asertiva. Se dijo que la asertividad no es un rasgo, y que un individuo actúa asertivamente o no dependiendo de las situaciones. Galassi y Galassi (1978) señalan que al evaluar una conducta como asertiva o no asertiva hay que tener siempre en cuenta tres aspectos: un aspecto conductual, un aspecto personal y un aspecto situacional. En el primero estarían comprendidas las conductas que ejecuta el individuo, como son: defender los derechos propios, iniciar y rechazar peticiones, hacer y recibir cumplidos, iniciar, mantener y terminar conversaciones, expresar amor y afecto, expresar opiniones personales incluyendo el no estar de acuerdo, y expresar ira y enfado justificados. El aspecto personal incluiría a las personas a quienes van dirigidas esas conductas, como son: amigos y conocidos (del mismo y distinto sexo), esposa/o o novia/o, padres y familia, figuras de autoridad, extraños y relaciones de negocios. El aspecto situacional incluiria las situaciones en que tendrian lugar las conductas, como por ejemplo privado/público, que sería demasiado largo de especificar. Al evaluar una conducta para clasificarla como asertiva o no asertiva habría que considerar entonces una 
combinación de los tres aspectos, conductas, situaciones, personas, de modo que la asertividad o no asertividad de la conducta de un sujeto pueda ser especificada situacionalmente al máximo.

\section{CARACTERISTICAS DEL INDIVIDUO QUE ACTUA ASERTIVAMENTE}

¿Qué características posee el individuo que actúa asertivamente en una gran variedad de situaciones? Este es un terreno en el que los diversos autores, al entrar en él, han expuesto muchas veces su filosofia particular sobre el individuo que ellos creen sería (idealmente) asertivo en la gran mayoría de las situaciones. Estos autores describen una serie de caracteristicas que piensan posee normalmente dicho individuo. Estas descripciones serian más que nada un apoyo moral para motivar al individuo no asertivo a cambiar su conducta por una más asertiva. Que el sujeto lo haga o no depende en último término de él mismo. Pero un ideal que motive su cambio puede ser una ayuda para un principio de cambio de conducta.

El individuo asertivo suele defenderse bien en sus relaciones interpersonales. Está satisfecho de su vida social y tiene confianza en sí mismo para cambiar cuando necesite hacerlo. $\mathrm{El}$ individuo asertivo es expresivo, espontáneo, seguro, y es capaz de influenciar y guiar a los otros (Galassi y cols., 1974). Fundamental para ser asertivo es el darse cuenta tanto respecto a sí mismo como al contexto que le rodea. El darse cuenta sobre uno mismo consiste en "mirar dentro» para saber lo que quiere antes de mirar alrededor para ver lo que los demás quieren y esperan de uno en una situación dada. Complementario a esto, está la capacidad de conocer las probables consecuencias de una conducta particular en un lugar determinado, y tomar la responsabilidad por esa consecuencia si escogemos actuar. "Mi propio interés es la primera cuestión en preguntar y explorar antes de que me preocupe por la consecuencia social. Habiendo delineado lo que quiero, debo ver luego si la consecuencia percibida de mi acción asertiva es demasiado penosa o arriesgada, está fuera de mi control realista, transgrede los derechos de otras personas o viola algún otro principio que tengo en gran estima» (Shoemaker y Satterfield, 1977, p. 55).

Salter (1949) dice que el individuo aşertivo actúa, sin pensar. Es directo. Cuando se enfrenta a un problema, toma de inmediato una acción constructiva. Le gusta la gente sinceramente, pero no le importa lo que puedan pensar. Habla de él mismo de manera no afectada. Toma decisiones rápidas y le gusta la responsabilidad. Sobre todo está libre de ansiedad. Es verdaderamente feliz (Salter, 1949).

El individuo asertivo está totalmente a cargo de sí mismo en las relaciones interpersonales, se siente capaz, sin engreimiento u hostilidad, es básicamente espontáneo en la expresión de los sentimientos y emociones, y es generalmente considerado y admirado por los otros (Alberti y Emmons, 1978). Esto último está en desacuerdo con lo que Salter (1949) pensaba del individuo asertivo, al que considera que es invariablemente subestimado por el no asertivo. Para este autor los criterios de la asertividad son la honestidad de la respuesta y el contenido de ella.

$\mathrm{La}$ asertividad tiende a ser autoreforzadora (Alberti y Emmons, 1978). Se supone que el aumentar la asertividad beneficia al sujeto de dos maneras significativas. Por un lado se piensa que comportarse de una manera más asertiva infundirá en el sujeto un mayor sentimiento de bienestar. Por otra parte se supone que el comportarse de una manera más asertiva hará que el sujeto sea más capaz de lograr recompensas sociales (así como materiales) significativas y así obtener más satisfacción de la vida (Rimm y Masters, 1974). Muy probables consecuencias del aumento de asertividad son los sentimientos de bienestar y alivio 
personal asi como una satisfacción interpersonal aumentada. Por otra parte la adquisición de las conductas asertivas conduce a una discriminación en las conductas incompatibles con la asertividad: agresividad y pasividad $\circ$ inhibición (Shoemaker y Satterfield, 1977). La asertividad intencional, activa, inhibe o reemplaza estos indeseables estilos de respuesta. Lazarus (1971) cree igualmente que el resultado de la conducta asertiva es una disminución de la ansiedad, unas relaciones más próximas y significativas, un respeto hacia uno mismo, y la adaptación social. Para Warren y Gilner (1978) la expresión de los sentimientos negativos, la defensa de los derechos propios y la expresión de opiniones permiten al individuo controlar el acceso directo a recompensas que pueden no haber estado disponibles anteriormente. Para Jakubowski (1977) la gente sana emocionalmente, que funciona totalmente, cree que puede producir un impacto efectivo en su ambiente. No sienten que sean víctimas desamparadas de los sucesos de la vida o de las exigencias de otras personas. Al contrario, se sienten responsables de ellas mismas porque creen que pueden entregarse a la conducta directa que afectará a los demás de forma constructiva.

Guerra y Taylor (1977) piensan que las personas asertivas creen en sus legítimos valores y sentimientos. Es sano y normal para ellas el tratar de que sus necesidades y deseos sean llenados por sus conocidos y amigos. Cada persona tiene muchas clases diferentes de necesidades que posiblemente no podrian satisfacerse por un único individuo. Cada persona tendría que tener tipos de amigos muy diferentes. La persona asertiva tiene conocidos, amigos y amante(s) capaces de llenar cada necesidad legítima que se pudiese esperar fuese llenada por otro individuo diferente del sujeto asertivo (Guerra y Taylor, 1977; Lazarus, 1971).

Finalmente bajo ciertas circunstancias, la utilidad personal de una aserción será de menor importancia que la utilidad de evitar la respuesta probable a esa aser- ción. Como señalan Alberti y Emmons (1978): «Es convicción nuestra que cada persona debería poder escoger cómo actuar. Si puedes actuar asertivamente bajo determinadas circunstancias, pero escoges no hacerlo, hemos cumplido nuestro objetivo... ("el de enseñar a la gente a ser asertiva"). Si al contrario eres incapaz de actuar asertivamente (por ejemplo, no puedes escoger cómo comportarte, sino que te acobardas con la no asertividad o estallas en la agresión) te gobernarán los demás y tu salud mental se resentirá. Nuestro criterio más importante para tu bienestar es que tú haces la elección" (p. 100).

\section{CONCLUSIONES}

A pesar de la gran popularidad alcanzada por el término "asertividad» y el "entrenamiento asertivo" en la década de los setenta, todavia no hay una definición universalmente aceptada. De igual manera, tampoco se han delimitado las dimensiones de la asertividad. Especificar qué es lo que comprende exactamente el constructo de la asertividad es algo que está por resolver. Aunque el entrenamiento asertivo es efectivo, el constructo teórico que lo abarca no está todavía establecido. Hay unas ideas generales, eso sí, normalmente aceptadas. Pero después cada autor añade otras ideas de su propia cosecha, en base a su experiencia clínica o a los estudios experimentales que haya realizado. Sin embargo ies necesaria una teoría de la asertividad? Lazarus (1971), por ejemplo, defiende la supremacia de las técnicas en la terapia de conducta, sin importarle mucho la teoría sobre ella. Lo importante es que las técnicas sean efectivas y ayuden a los pacientes. Hay técnicas que funcionan y muy bien, pero que no se han deducido de una teoría general. Simplemente se sabe que funcionan. ¿Podríamos decir lo mismo respecto al entrenamiento asertivo? Se sabe que éste es efectivo con sujetos que padecen problemas interpersonales. ¿Es necesaria una 
teoria de la asertividad? Se podría decir que sería conveniente, que vendria bien, pero que no resulta fundamental. Sobre todo a nivel práctico. El entrenamiento asertivo funciona sin una teoría establecida de la asertividad. Importa que el entrenamiento sea efectivo, que ayude a los pacientes. ¿Para qué sirve una teoria si no se lleva a la práctica? La efectividad del entrenamiento asertivo está fuera de duda. Si se establece una teoría general que lo abarque será bienvenida. Pero mientras tanto el entrenamiento asertivo debe continuar ayudando a la gente a funcionar mejor socialmente.

Se puede decir sin embargo, que, aunque ninguna definición actual es suficientemente comprensiva para representar adecuadamente el trabajo diverso en el área, hay sin embargo un acuerdo bastante amplio sobre la concepción general de la conducta asertiva. Así, con Bellack (1979) se puede decir que parece haber cuatro elementos que son comunes a la mayoría de las definiciones:

1. La ejecución de las situaciones interpersonales depende de una serie de diferentes componentes de respuesta verbales y no-verbales.
2. Los parámetros particulares que comprende la conducta adecuada y su configuración varían según la situación. La conducta asertiva es específica situacionalmente.

3. Los variados elementos componentes que comprende la conducta social adecuada son habilidades de respuesta aprendidas. Los individuos pueden variar en su nivel general de la conducta asertiva, así como en sus habilidades en diferentes situaciones especificas.

4. Cuando se pueden identificar déficit en conductas sociales específicas, pueden ser puestos como objetivos y remediados por el entrenamiento.

Finalmente, añadir que parece darse un abandono del vocablo "asertividad" por la expresión "habilidades sociales", aunque el concepto es el mismo. Esto no tendria mayor importancia que el que los profesionales se pusieran de acuerdo y decidiesen qué término emplear. Aunque las espadas están en alto, parece ser que el término más idóneo de «habilidades sociales" se va imponiendo.

\section{Resumen}

En este articulo se revisan diferentes definiciones y dimensiones que se ban dado sobre la asertividad, asi como también se bosquejan las raices de este concepto y la tendencia actual que existe entre los profesionales para usar otras expresiones en sustitución del término "rasertividad». No bay ninguna definición que baya sido universalmente aceptada, ni un grupo de dimensiones que se bayan establecido definitivamente. Se revisan una serie de caracteristicas que diferentes aufores atriburyen al individuo asertivo y, finalmente se especifican unos elementos que son comunes a la mayoría de las definiciones de la conducta asertiva.

\section{Summary}

This article is concerned with the concept of assertiveness. The roots, substitutions with otber terms, definitions and dimensions bave been revieved. Not one definition has been universally accepted, thougb an implicit agreement about the meaning of the construct of assertiveness in seen among the clinicians and experimenters. The assertive behavior is divided into several dimensions, but these ones bave not yet been vell establisbed, thougb the assertive training is a successful behavior therapy technique of treatment. Finally, some cbaracteristics of the assertive bebavior that are recognized in general by professionals in the field are stated. 


\title{
Resumé
}

\begin{abstract}
Dans cet article on trate le tbème de rassertivité. On a revisé quelques définitions et dimensions du comportement assertif et aussi on a vu les origines de ce concept et son cbangement pour autres noms dans ce moment actuel. Aucune définition a été universellement accepté, non plus on été établi les dimensions exactes de ce concept. Aussi on voie quelques caractéristiques de rindividu assertif et, finalement, on a spécifié quelques élements qui sont commens pour la majorite des definitions du comportement assertif.
\end{abstract}

\section{Notas}

1 Curran, J. P.: Social skills training manual. Unpublisbed manuscript. Brown Medical School/Veterans Administration Hospital, 1977.

\section{Referencias}

Albertl, R. E.: Assertive behavior training: definitions, overview, contributions. In R. E. Alberti (ed.), Assertiveness: Innovations, Applications, Issues. San Luis Obispo, California: Impact, 1977(a).

Albert, R. E.: Issues in assertive behavior training. In R. E. Alberti (ed.), Assertiveness: Innovations, Aplications, Issues. San Luis Obispo, California: Impact, 1977(b).

Alberti, R. E. y Emmons, M. L.: Your Perfect Rigbt: A Guide to Assertive Bebavior. San Luis Obispo, California: Impact, 1978 (3rd edition).

Alberti, R. E., Emmons, M. L., Fodor, I. G., Galassi, J. P., Galassi, M. D., Garnett, L., JakuBOWSKI, P. y WOLFE, J. L.: A statement of «Principles for ethical practice of assertive behavior training». In R. E. Alberti (ed.), Assertiveness: Innovations, Aplications, Issues. San Luis Obispo, California: Impact, 1977.

BellaCK, A. S.: Behavioral assessment of social skills. In A. S. Bellack and M. Hersen (eds.), Researcb and Practice in Social Skills Training. New York: Plenum Press, 1979.

Bornstein, P. H., Bellack, A. S. y Hersen, M.: Social skills training for unassertive children: A multiple baseline analysis. Journal of Applied Bebavior Analysis, 1977, 10, 183-195.

Brown, S. D. y BROWN, L. W.: Trends in assertion training research and practice: A content analysis of the published literature. Journal of Clinical Psycbology, 1980, 36, 265-269.

Curran, J. P.: Social skills: Methodological issues and future directions. In A. S. Bellack and M. Hersen (eds.), Research and Practice in Social Skills Training. New York: Plenum Press, 1979.

EISLer, R. M. y Frederiksen, L. W.: Perfecting Social Skills: A Guide to Interpersonal Bebavior Development. New York: Plenum Press, 1980.

FENSTERhEIM, H. y BAER, J.: No diga Si cuando quiera decir No. Barcelona: Grijalbo, 1976.

Galassi, J. P., Delo, J. S., Galassi, M. D. y Bastien, S.: The college self expression scale: A measure of assertiveness. Bebavior Therapy, 1974, 5, 165-171.

Galassi, J. P. y Galassi, M. D.: Assessment procedures for assertive behavior. In R. E. Alberti (ed.), Assertiveness: Innovations, Aplications, Issues. San Luis Obispo, California: Impact, 1977.

Galassi, J. P. y Galassı, M. D.: Modification of heterosocial skills deficits. In A. S. Bellack and M. Hersen (eds.), Research and Practice in Social Skills Training. New York: Plenum Press, 1979.

Galassi, M. D. y Galassi, J. P.: Assertion: A critical review. Psycbotherapy: Theory, Research and Practice, 1978, 15, 16-29.

GAMBrILL, E. D. y RICHEY, C. A.: An assertion inventory for use in assessment and research. Bebavior The rapy, $1975,6,550-561$.

Guerra, J. J. y TAYlor, P. A.: The four assertive myths: A fable. In R. E. Alberti (ed.), Assertiveness: Innovations, Applications, Issues. San Luis Obispo, California: Impact, 1977.

Heinberg, R. G., Montgomery, D., Madsen, C. H. jr. y Heimberg, J. S.: Assertion training: A review of the literature. Bebavior Tberapy, 1977, 8, 953-971.

Jakubowskı, P. A.: Assertive behavior and clinical problems of women. In R. E. Alberti (ed.), Assertiveness: Innovations, Aplications, Issues. San Luis Obispo, California: Impact, 1977.

Kelley, C.: Assertion Training: A Facilitator's Guide. San Diego, California: University Associates, 1979.

LAzARUS, A. A.: Behavior rehearsal vs. non-directive therapy vs. advice in effectiong behaviour change. Bebaviour Researcb and Tberapy, 1966, 4, 209-212.

Lazarus, A. A.: Bebavior Therapy and Beyond. New York: McGraw-Hill, 1971. 
LAZARUS, A. A.: On assertive behavior: A brief note. Bebavior Tberapy, 1973, 4, 697-699.

LORR, M. y MORE, W. W.: Personal Relations Inventory, 1979.

LORR, M. y MORE, W. W.: Four dimensions of assertiveness. Multivariate Bebavioral Research, 1980, 2, $127-138$.

LORR, M., MORE, W. W. y MANSUETO, C. S.: The structure of assertiveness: A confirmatory study. Bebaviour Research and Therapy, 1981, 19, 153-156.

MAC Donald, M. L.: Measuring assertion: A model and method. Bebavior Therapy, 1978, 9, 889-899.

MATSON, J. L., KAZDIN, A. E. y EsveldT-DAwSON, K.: Training interpersonal skills among mentally retarded and socially dysfunctional children. Behavior Researcb and Therapy, 1980, 18, 419-427.

Rathus, S. A., Fox, J. A. y Cristofaro, J. D.: Perceuved structure of aggresive and assertive behaviors. Psychological Reports, 1979, 44, 695-698.

RICH, A. R. y SCHROEDER, H. E.: Research issues in assertiveness training. Psycbological Bulletin, 1976, 83, 1081-1096.

RimM, D. C. y MASTERS, J. C.: Behavior Therapy: Tecbniques and Empirical Findings. New York: Academic Press, 1974.

Romano, J. M. y Bellack, A. S.: Social validation of a component model of assertive behavior. Journal of Counsulting and Clinical Pycbology, 1980, 48, 478-490.

SAlter, A.: Conditioned Reflex Therapy. New York: Farrar, Starus and Giroux, 1949.

ShOEMAKER, M. E. y SATTERFIELD, D. O.: Assertion training: An identity crisis that's coming on strong. In R. E. Alberti (ed.), Assertiveness: Innovations, Applications, Isswes. San Luis Obispo: California: Impact, 1977.

Smith, M. J.: Cuando digo No me siento culpable. Barcelona: Grijalbo, 1977.

TROWER, P.: Fundamentals of interpersonal behavior: A social-psychological perspective. In A. S. Bellack and M. Hersen (eds.), Researcb and Practice in Social Skills Training. New Yowk: Plenum Press, 1979.

Trower, P., Bryant, B. y ARgYle, M.: Social Skills \& Mental Healtb. London: Methuen, 1978.

TURNER, S. M. y ADAMs, H. E.: Effects of assertive training on three dimensions of assertiveness. Bebaviour Research and Therapy, 1977, 15, 475-483.

Van Hasselt, V. B., Hersen, M., Whitehill, M. B. y Bellack, A. S.: Social skill assessment and training for children: An evalutive review. Bebaviosr Researeb and Tberapy, 1979, 17, 413-437.

WARREN, N. J. y GILNER, F. H.: Measurement of positive assertive behaviors: The behavioral test of tenderness expression. Behavior Therapy, 1978, 9, 178-184.

WOLPE, J.: La práctica de la terapia de conducta. México: Trillas, 1977. 